\title{
Avaliação da Velocidade Média na Aorta Torácica Descendente em Fetos com Anemia
}

\author{
Measurement of the Mean Blood Velocity in the Descending \\ Thoracic Aorta in Anemic Fetuses
}

Marcos Roberto Taveira, Antônio Carlos Vieira Cabral, Henrique Vitor Leite Ana Paula Brum, Alim Alves Demian, Isabela Gomes de Melo

\begin{abstract}
RESUMO
Objetivo: verificar se existe correlação significativa entre a velocidade média na dopplerfluxometria da artéria aorta torácica descendente e o grau de anemia fetal.

Métodos: estudo prospectivo, transversal, no qual foram analisados 66 fetos de gestantes isoimunizadas, em que se realizou a cordocentese para a realização de transfusões intrauterinas pela via intravascular (66,7\%). Nos fetos que foram submetidos à transfusão intrauterina pela via intraperitoneal, ou naqueles casos em que não houve necessidade de tratamento intra-uterino (33,3\%), a determinação da concentração de hemoglobina do cordão foi realizada pela punção do cordão umbilical, no momento da interrupção da gestação. Neste grupo de fetos estudados, foi realizado exame dopplerfluxométrico da artéria aorta torácica descendente, sendo calculada a velocidade média de fluxo. Foi realizado estudo de associação entre as variáveis. Foram também calculados os valores de sensibilidade, especificidade, valores preditivos positivo e negativo.

Resultados: observou-se correlação significativa e inversa entre a velocidade média na artéria aorta torácica descendente e o nivel de hemoglobina fetal. A velocidade média na dopplerfluxometria da artéria aorta torácica descendente apresentou sensibilidade de 47,5\% para anemia fetal moderada $(\mathrm{Hg}<10 \mathrm{~g} / \mathrm{dL})$, com o teste exato de Fisher apresentando valor de $p<0,01$, e de 54,5\% para anemia fetal grave $(\mathrm{Hg}<7,0 \mathrm{~g} / \mathrm{dL})$, com um valor de $p=0,01$.

Conclusões: houve associação significativa entre a velocidade média na aorta torácica descendente e o grau de diagnóstico de anemia fetal.
\end{abstract}

PALAVRAS-CHAVE: Isoimunização. Dopplerfluxometria. Anemia fetal. Cordocentese.

\section{Introdução}

A isoimunização materna pelo fator Rh permanece ainda hoje como importante causa de anemia fetal. Há anos que se utilizam métodos invasivos para determinação do grau de anemia fetal, inicialmente a amniocentese para a dosagem de bilirrubina no líquido amniótico ${ }^{1}$, e atualmente tem-se recorrido à cordocentese para obtenção do sangue fetal e assim determinar o nível

Centro de Medicina Fetal do Hospital das Clínicas da Universidade de Minas Gerais

Correspondência:

Marcos Roberto Taveira

MedFet - Centro Mineiro de Medicina Fetal

Rua dos Otoni, 909, sala 2105, Bairro Santa Efigênia

30150-270 - Belo Horizonte - MG de hemoglobina e outros indices hematimétri$\cos ^{2,3}$.

Muitos estudos realizados nas últimas décadas têm buscado identificar métodos propedêuticos não-invasivos que possam seguramente identificar, e se possivel graduar, o quadro de anemia fetal. Inicialmente a cardiotocografia foi utilizada $^{4,5}$ mas o seu real valor ainda está sendo determinado. A seguir se tentou a ecografia fetal com a mesma finalidade ${ }^{6}$, valorizando principalmente as alterações morfológicas do feto, placenta e volume do líquido amniótico. Porém, nenhum dos métodos citados apresentou segurança ou valor preditivo que pudesse tornar desnecessário o uso dos métodos invasivos. Recentemente alguns autores têm utilizado a técnica da dopplerfluxo- 
metria com o intuito de também avaliar este método, buscando encontrar a correlação das suas alterações com a anemia fetal.

Nas gestantes isoimunizadas, o feto, quando Rh positivo, sofre anemia progressiva, conforme a carga de anticorpos maternos originando estado hiperdinâmico compensatório secundário à redução na viscosidade sangüinea e em resposta à hemólise fetal. Rightmire et al. ${ }^{7}$ observaram que nestas situações ocorre um aumento na velocidade média (Vmed) da artéria aorta torácica descendente (AAo), quando estudaram um grupo de 21 fetos de gestantes isoimunizadas, e este achado correlacionou-se de maneira significativa e inversa com o seu hematócrito.

Nicolaides et al. ${ }^{8}$ estudaram 68 gestantes isoimunizadas, por meio da dopplerfluxometria da AAo, insonada no nível do diafragma fetal. Observaram correlação significativa e inversa entre o aumento na Vmed na AAo e o déficit de hemoglobina nos fetos não-hidrópicos. Este achado refletiria aumento no fluxo sangüineo devido a aumento no débito cardiaco fetal. Nos fetos hidrópicos, observou-se redução na Vmed na dopplerfluxometria da AAo, sugerindo uma fase de descompensação cardiaca. Neste estudo a Vmed na dopplerfluxometria da AAo apresentou sensibilidade de $53 \%$ para o diagnóstico da anemia fetal grave $^{8}$.

Hecher et al. ${ }^{9}$ avaliaram a dopplerfluxometria da aorta descendente, veia cava inferior, ducto venoso e artéria cerebral média (ACM) e o fluxo das válvulas atrioventriculares, na detecção da anemia fetal em um grupo de 38 fetos de gestantes isoimunizadas. Os autores observaram que nos fetos não-hidrópicos houve aumento significativo da velocidade média do fluxo no ducto venoso, aorta torácica descendente e ACM, caracterizando estado hiperdinâmico, secundário à anemia fetal ${ }^{9}$.

O objetivo deste estudo é avaliar a associação entre a Vmed de fluxo na dopplerfluxometria da AAo com os niveis de hemoglobina fetal.

\section{Pacientes e Métodos}

Este é um estudo prospectivo em que, no período de janeiro de 1998 a setembro de 2000, foram estudados 66 fetos de gestantes isoimunizadas. As pacientes isoimunizadas acompanhadas em nosso serviço, quando da sua matrícula, são informadas do protocolo de acompanhamento (aprovado previamente pela Comissão de Ética), quanto aos riscos dos procedimentos invasivos e quan- do concordavam, assinavam o termo de consentimento.

Foram selecionados 66 fetos de gestação única sendo que 44 casos $(66,7 \%)$ foram submetidos à cordocentese para a realização de transfusões intra-uterinas e simultaneamente foi determinada a concentração da hemoglobina fetal. Os 22 casos restantes $(33,3 \%)$ não foram submetidos à cordocentese para transfusão, e quando esta se fez necessária foi utilizada a via intraperitoneal. Neste grupo o sangue fetal foi obtido puncionandose o cordão umbilical, para a determinação da concentração de hemoglobina por ocasião do parto, no momento em que se realizava a clampagem do funículo. Em todos os casos realizou-se previamente, em intervalo sempre inferior a 24 horas antes do procedimento, exame dopplerfluxométrico da AAo.

A indicação para a realização da cordocentese foi baseada na presença de teste de Coombs indireto com titulação igual ou superior a $1 / 16$, em painel de hemáceas revelando presença de antígenos eritrocitários e no passado obstétrico. A técnica da punção do cordão umbilical utilizada neste estudo foi a mesma descrita por Daffos et al. ${ }^{10}$, utilizando o auxílio ecográfico. Os niveis de hemoglobina foram determinados pelo uso do aparelho Hemocue ${ }^{\circledR}$.

O exame dopplerfluxométrico da AAo foi realizado por um único examinador, com a paciente em posição de semi-Fowler, estando o feto em apnéia e na ausência de movimentos corporais. Utilizou-se filtro acústico de $100 \mathrm{~Hz}$. A medida da Vmed na AAo foi obtida após insonação deste vaso em seu trajeto no nivel do diafragma fetal. O fluxograma foi obtido após a observação de pelo menos cinco ciclos cardíacos uniformes e homogêneos, sendo calculada a média aritmética entre pelo menos três ciclos.

Foi utilizada, como índice dopplerfluxométrico, a velocidade média de fluxo na AAo. O ângulo entre o transdutor do aparelho de dopplerfluxometria e a direção do fluxo na AAo foi corrigido para permanecer sempre o mais próximo de zero. $\mathrm{O}$ exame foi realizado em aparelho Sonoace 8800 da Medson e também no aparelho Acustic Image 5500 S Envision, da marca Dornier.

Foram excluídos do estudo todos os casos em que a dopplerfluxometria da artéria aorta torácica descendente foi realizada em um periodo superior a 24 horas antecedendo as transfusões intrauterinas ou a interrupção da gestação.

Realizou-se a associação entre a velocidade média de fluxo no exame de dopplerfluxometria da artéria aorta torácica descendente e os diversos niveis de hemoglobina fetal obtida pela cordocentese. Utilizamos como parâmetros de normalida- 
de para a Vmed na AAo a curva de normalidade proposta por Nicolaides et al. ${ }^{8}$. Valores alterados eram aqueles superiores à dois desvios padrão acima da média.

Os resultados foram estratificados em dois grupos: um grupo na qual a hemoglobina de cordão apresentava valores menores que de $7,0 \mathrm{~g} / \mathrm{dL}$ e um segundo estrato em que a taxa de hemoglobina fetal foi inferior a 10,0 g/dL. Procuramos estudar se a velocidade média na AAo apresentou correlação significativa para a detecção dos dois niveis de anemia fetal, por serem estes os níveis já tradicionalmente definidos como anemia fetal grave e moderada, respectivamente ${ }^{2}$.

Para a análise estatística utilizamos o método do $\chi^{2}$, aceitando como significativo um valor de $\mathrm{p}<0,05$; calculamos também a sensibilidade, especificidade e valores preditivos da Vmed na AAo. Realizamos também a análise descritiva das variáveis, calculando valores de média, mediana, máxima e mínima, nesta amostra, por meio do software Minitab 2000.

\section{Resultados}

Foram avaliados 66 casos nos quais foi realizada a cordocentese para a realização das transfusões intravasculares, quando indicadas $(n=44)$, e nos demais casos puncionou-se o cordão umbilical, no momento do parto, para se conhecer a concentração de hemoglobina fetal $(n=22)$.

A idade gestacional média neste estudo foi de 30,7 semanas e a mediana foi de 31 semanas. A idade gestacional minima em que foi realizada a punção do cordão umbilical para pesquisa da anemia fetal foi 20,5 semanas, e a máxima foi de 40,4 semanas.

O nível médio da $\mathrm{Hg}$ de cordão foi de $11,5 \mathrm{~g} / \mathrm{dL}$ (mediana de 12,0 g\%), com valor mínimo de 3,3 g/dL e máximo de $17,1 \mathrm{~g} / \mathrm{dL}$.

Para a variável Vmed na AAo, o valor médio foi de $21,8 \mathrm{~cm} / \mathrm{s}$, com valor mínimo de 10,0 e máximo de $41,4 \mathrm{~cm} / \mathrm{seg}$, a mediana de $21,8 \mathrm{~cm} / \mathrm{s}$.

Houve uma associação inversa estatisticamente significante entre a velocidade média na artéria aorta torácica descendente e o achado de hemoglobina de cordão menor ou igual a $10,0 \mathrm{~g} / \mathrm{dL}$.

A velocidade média na dopplerfluxometria da artéria aorta torácica descendente apresentou sensibilidade de 47,4\% na detecção de hemoglobina de cordão menor ou igual a 10,0 g/dL.

A Tabela 1 apresenta os resultados do grupo com nivel de hemoglobina de cordão menor ou igual a $10,0 \mathrm{~g} / \mathrm{dL}$ e sua correlação com a dopplerfluxometria de artéria aorta, alterada ou não.
Tabela 1 - Correlação entre Vmed na artéria aorta torácica descendente e a concentração de hemoglobina de cordão no grupo com niveis de $\mathrm{Hg}$ fetal inferiores a $10,0 \mathrm{~g} / \mathrm{dL}$.

\begin{tabular}{lccc}
\hline & \multicolumn{2}{c}{ Vmed AAo } & \\
& Alterada & Normal & Total \\
\hline $\mathrm{Hg}<10 \mathrm{~g} / \mathrm{dL}$ & 9 & 10 & 19 \\
$\mathrm{Hg}>10 \mathrm{~g} / \mathrm{dL}$ & 4 & 43 & 47 \\
Total & 13 & 53 & 66
\end{tabular}

Vmed- Velocidade média AAo- Artéria aorta torácica descendente

Sensibilidade $=69,2 \%$

Especificidade $=81,1 \%$

VPP $=47,4 \%$

VPN $=91,5 \%$

$\chi^{2}=12,92$, valor de $p<0,0003$

Teste exato de Fisher - valor de $p=0,0008$

A Vmed na dopplerfluxometria da AAo também mostrou correlação estatisticamente significante com niveis de hemoglobina detectados na cordocentese menores ou iguais a $7,0 \mathrm{~g} / \mathrm{dL}$, $\left(\chi^{2}=6,60, p<0,01\right)$.

Para niveis de hemoglobina fetal menores ou iguais a $7,0 \mathrm{~g} / \mathrm{dL}$, a sensibilidade da Vmed na dopplerfluxometria da AAo foi de $54,5 \%$.

Na Tabela 2 apresentamos o resultado obtido no grupo com hemoglobina fetal menor ou igual a $7,0 \mathrm{~g} / \mathrm{dL}$.

Tabela 2 - Correlação entre a Vmed na dopplerfluxometria da AAo e a concentração de hemoglobina do cordão no grupo de fetos com níveis de $\mathrm{Hg}$ fetal inferiores a 7,0 g/dL.

\begin{tabular}{lccc}
\hline & \multicolumn{2}{c}{ Vmed AAo } & \\
& Alterada & Normal & Total \\
\hline $\mathrm{Hg}<7,0 \mathrm{~g} / \mathrm{dL}$ & 6 & 5 & 11 \\
$\mathrm{Hg}>7,0 \mathrm{~g} / \mathrm{dL}$ & 10 & 45 & 55 \\
Total & 16 & 50 & 66
\end{tabular}

$\mathrm{V}$ med- velocidade média

Sensibilidade $=37,5$

AAo- Artéria aorta torácica descendente

Especificidade $=90,0 \%$

$\mathrm{VPP}=54,5 \%$

VPN $=81,8 \%$

$\chi^{2}=6,60$ $p<0,01$

\section{Discussão}

O manuseio da isoimunização materna pelo fator $\mathrm{Rh}$ e também pelos antígenos irregulares requer diagnóstico preciso da sensibilização assim como a tentativa de se determinar a gravidade do processo, para que possamos identificar quais os fetos que estão expostos a riscos de uma anemia grave, necessitando de técnicas invasivas, propedêutica ou terapêuticas.

Inicialmente, o diagnóstico da sensibilização é realizado por meio do teste de Coombs indireto, 
e paralelamente realiza-se o painel de hemáceas com o objetivo de se identificar qual antígeno específico é responsável pela sensibilização, e assim determinar seu potencial de hemólise.

A amniocentese para espectrofotometria do líquido amniótico nos permite estimar a concentração de bilirrubina pela medida da diferença de densidade ótica no comprimento de onda $450 \mathrm{~nm}$, e indiretamente determinar o grau de hemólise fetal.

$\mathrm{O}$ acesso direto ao sangue fetal, por meio da cordocentese, permite a determinação do grupo sangüineo e fator Rh do feto, e também a determinação da sua concentração de hemoglobina e do hematócrito, detectando com maior acurácia o grau de anemia. Entretanto, a punção do cordão umbilical se associada a niveis inaceitáveis de hemorragia fetomaterna (50\%), tornando-a assim um método impróprio para uso universal no diagnóstico do nível de comprometimento fetal na isoimunização $\mathrm{Rh}$, apesar de ser a primeira opção no tratamento intra-uterino desta doença ${ }^{11}$.

Dos métodos não-invasivos estudados para o diagnóstico da isoimunização $\mathrm{Rh}$, a dopplerfluxometria tem se mostrado ser o mais promissor.

Nesse estudo, encontramos associação significativa e inversa entre o aumento da Vmed da AAo e a anemia fetal. Este resultado pode ser interpretado como uma resposta de adaptação fetal secundária à hemólise. Esses dados sugerem que em fetos anêmicos ocorre aumento na velocidade média de fluxo na AAo, caracterizando estado circulatório hiperdinâmico, em resposta à redução na viscosidade sangüinea resultante da anemia fetal. $\mathrm{O}$ aumento na velocidade de fluxo sangüineo arterial provavelmente reflete aumento no volume sistólico, levando a aumento no fluxo sangüíneo periférico, para manter perfusão adequada aos órgãos. Esses nossos resultados estão de acordo com alguns outros citados por diversos autores ${ }^{7-9}$.

Em nosso estudo, procuramos estratificar os resultados de acordo com o grau de anemia fetal, desta forma estudamos a associação da Vmed na AAo e a concentração de hemoglobina fetal em dois niveis distintos: hemoglobina de cordão $<10,0 \mathrm{~g} / \mathrm{dL}$ e $<7,0 \mathrm{~g} / \mathrm{dL}$, conforme definição de anemia fetal moderada e grave, respectivamente. Houve correlação estatisticamente significativa para os dois niveis de anemia fetal pré-definidos. Para o grupo com hemoglobina fetal $<10,0 \mathrm{~g} / \mathrm{dL}$, a sensibilidade da Vmed na AAo foi de 47,4\% e a especificidade de $91,5 \%$. Entretanto, para o grupo com anemia fetal grave, a sensibilidade foi menor, de $54,5 \%$, mantendo uma especificidade muito boa, de $81,8 \%$.

$\mathrm{Na}$ gestação normal há aumento progressivo na Vmed de fluxo na AAo, até a $32^{\mathrm{a}}$ semana de gravidez, quando então permanece constante; este aumento visa atender a maiores demandas do feto em crescimento, em contraste com um aumento linear e progressivo na velocidade de fluxo na artéria carótida comum, sugerindo que maior percentual de fluxo seja dirigido ao sistema nervoso central, para compensar a queda progressiva na $\mathrm{PO}_{2}{ }^{12}$.

Nos fetos anêmicos, não-hidrópicos, em estágios iniciais de anemia, ocorre redução na viscosidade sangüínea, em função da queda do hematócrito. Conseqüentemente, há resposta circulatória no concepto, gerando estado hiperdinâmico de fluxo, resultando em aumento na velocidade média detectado na dopplerfluxometria da artéria aorta torácica descendente, visando manter constante e intacto o aporte de oxigênio tecidual, já tendo sido comprovado tanto em estudos clínicos como em estudos experimentais ${ }^{13}$.

Nos casos de anemia fetal grave, com niveis de hemoglobina $<7,0 \mathrm{~g} / \mathrm{dL}$, poderia haver queda na velocidade média de fluxo detectada na dopplerfluxometria da artéria aorta torácica descendente, como resultado de redução na contratilidade do miocárdio, sugerindo o início de um estágio de descompensação fetal, com insuficiência cardíaca franca ${ }^{8}$.

Em nosso estudo, encontramos 11 casos em que os niveis de hemoglobina no cordão eram inferiores a $7,0 \mathrm{~g} / \mathrm{dL}$, sendo que todos eles apresentavam hidropisia fetal por ocasião da primeira transfusão intra-uterina. Em cinco casos conseguiu-se a reversão da hidropisia fetal por meio da terapia fetal com transfusões intra-uterinas seriadas, com sobrevida perinatal de todos estes conceptos. Nos seis casos restantes, os fetos evoluíram para o óbito, indicando comprometimento fetal grave, sugerindo que parte destes conceptos (o grupo que evoluiu para óbito intra-uterino) já poderia apresentar algum grau de insuficiência cardíaca.

De acordo com os resultados obtidos neste estudo, o achado de aumento na Vmed na dopplerfluxometria de AAo, em fetos de gestantes sensibilizadas, sugere a presença de anemia fetal importante, moderada ou grave. Desta forma, a dopplerfluxometria da aorta pode nos fornecer dados a respeito da necessidade de uma nova transfusão intra-uterina, constituindo assim um método não-invasivo de grande valor no acompanhamento destes casos.

O alto valor de especificidade da Vmed na AAo nos permite afirmar que estes fetos apresentam seguramente niveis de hemoglobina superiores a $10,0 \mathrm{~g} / \mathrm{dL}$, sendo importante instrumento de acompanhamento das gestantes isoimunizadas. Existe associação significativa e inversa entre a velocidade média de fluxo na artéria aorta torácica descendente e o nivel de hemoglobina fetal. 


\section{SUMMARY}

Purpose: to verify if there is an association between the mean blood velocity in the descending thoracic aorta and fetal anemia diagnosis.

Methods: this is a prospective, cross-sectional study in which the mean blood velocities in the fetal aorta, in 66 fetuses at risk for severe anemia due to severe $R h$ immunization, and cord blood hemoglobin levels were analyzed comparatively. The hemoglobin level was obtained by cordocentesis if an intravascular transfusion was performed for severe anemia, however, if the fetus received an intrauterine transfusion by the intraperitoneal route or if the fetus did not receive a transfusion at all, hemoglobin level was measured at the time of pregnancy termination by umbilical cord puncture. The authors made a statistical association between the mean blood velocity in fetal descending thoracic aorta and the diagnosis offetal anemia. The $\chi^{2}$ test was used for statistical analysis and a $p$ value <0,05 was used to indicate significance.

Results: there was a significant and indirect association between the mean blood velocity in the descending thoracic aorta and the detection of fetal anemia. The mean blood velocity in fetal thoracic aorta had a sensitivity of $47.4 \%$ for the diagnosis of moderate fetal anemia $(\mathrm{Hg}<10.0 \mathrm{~g} / \mathrm{dL})$, with a $p$ value $<0.01$ by the Fisher exact test, and a sensitivity of $54.5 \%$ for severe Rh isoimmunization $(\mathrm{Hg}<7.0 \mathrm{~g} / \mathrm{dL})$, with a $p$ value $=0.01$.

Conclusion: this study revealed a significant indirect correlation between mean blood velocity in the descending thoracic aorta and the detection of fetal anemia due to Rh isoimmunization.

KEY WORDS: Isoimmunization. Doppler flowmetry. Cordocentesis. Fetal anemia.

\section{Referências}

1. Liley AW. Liquor amni analysis in management of the pregnancy complicated by rhesus sensitization. Am J Obstet Gynecol 1961; 82:1359-70.

2. Cabral ACV, Pereira KA, Assreuy SS, Taveira MR. Isoimunização materna e doença hemolítica perinatal. Realidade e perspectivas. J Bras Ginecol 1998; 108:181-5.
3. Weiner CP, Williamson RA, Wenstrom KD, et al. Management of fetal hemolytic disease by cordocentesis. 2. Outcome and treatment. Am J Obstet Gynecol 1991; 165:1302-7.

4. Visser GH. Antepartum sinusoidal and decelerative heart rate patterns in $\mathrm{Rh}$ disease. Am J Obstet Gynecol 1982; 143:538-44.

5. Corrêa MD, Cabral ACV, Lima MIM. Cardiotocografia no seguimento de fetos submetidos à transfusão intra-uterina. J Bras Ginecol 1987; 97:381-4.

6. Nicolaides KH, Fontanarosa M, Gabbe SG, Rodeck $\mathrm{CH}$. Failure of ultrasonographic parameters to predict the severity of fetal anemia in rhesus isoimmunization. Am J Obstet Gynecol 1988; 158:920-6.

7. Rightmire DA, Nicolaides $\mathrm{KH}$, Rodeck $\mathrm{CH}$, Campbell $\mathrm{S}$. Fetal blood velocities in $\mathrm{Rh}$ isoimmunization: relationship to gestational age and to fetal hematocrit. Obstet Gynecol 1986; 68:233-6.

8. Nicolaides KH, Bilardo CM, Campbell S. Prediction of fetal anemia by measurement of the mean blood velocity in the fetal aorta. Am J Obstet Gynecol 1990; 162:209-12.

9. Hecher K, Snijders R, Campbell S, Nicolaides KH. Fetal venous, arterial, and intracardiac blood flows in red blood cell isoimmunization. Obstet Gynecol 1995; 85:122-8.

10.Daffos F, Capella-Pavlovsky M, Forestier F. Fetal blood sampling during pregnancy with use of a needle guided by ultrasound: a study of 606 consecutive cases. Am J Obstet Gynecol 1985; 153:655-60.

11.Bowman JM, Pollock JM, Peterson LE, Harman CR, Manning FA, Menticoglou SM. Fetomaternal hemorrhage following funipuncture: increase in severity of maternal red-cell alloimmunization. Obstet Gynecol 1994; 84:839-43.

12. Copel JA, Grannum PA, Green JJ, Belanger K, Hobbins JC. Pulsed Doppler flow-velocity waveforms in the prediction of fetal hematocrit of the severely isoimmunized pregnancy. Am J Obstet Gynecol 1989; 161:341-4.

13.Huikeshoven FJ, Hope ID, Power GG, Gilbert RD, Longo LD. A comparison of sheep and human fetal oxygen delivery systems with use of a mathematical model. Am J Obstet Gynecol 1985; 151:449-55. 